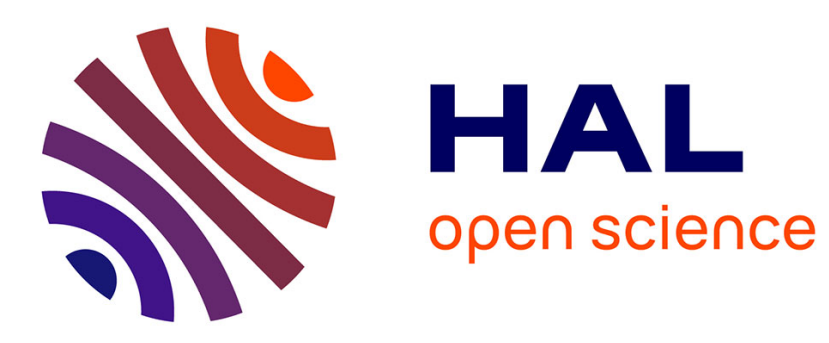

\title{
The Folding Pathway of the KIX Domain
}

Francesca Troilo, Daniela Bonetti, Angelo Toto, Lorenzo Visconti, Maurizio Brunori, Sonia Longhi, Stefano Gianni

\section{To cite this version:}

Francesca Troilo, Daniela Bonetti, Angelo Toto, Lorenzo Visconti, Maurizio Brunori, et al.. The Folding Pathway of the KIX Domain. ACS Chemical Biology, 2017, 12 (6), pp.1683-1690. 10.1021/acschembio.7b00289 . hal-03499989

\section{HAL Id: hal-03499989 \\ https://hal.science/hal-03499989}

Submitted on 5 Jan 2022

HAL is a multi-disciplinary open access archive for the deposit and dissemination of scientific research documents, whether they are published or not. The documents may come from teaching and research institutions in France or abroad, or from public or private research centers.
L'archive ouverte pluridisciplinaire HAL, est destinée au dépôt et à la diffusion de documents scientifiques de niveau recherche, publiés ou non, émanant des établissements d'enseignement et de recherche français ou étrangers, des laboratoires publics ou privés. 


\section{The folding pathway of the KIX domain}

Francesca Troilo ${ }^{1,2}$, Daniela Bonetti ${ }^{1}$, Angelo Toto ${ }^{1}$, Lorenzo Visconti ${ }^{1}$, Maurizio Brunori $^{1}$, Sonia Longhi ${ }^{2}$, and Stefano Gianni ${ }^{1 *}$

${ }^{1}$ Istituto Pasteur - Fondazione Cenci Bolognetti, Dipartimento di Scienze Biochimiche “A. Rossi Fanelli” and Istituto di Biologia e Patologia Molecolari del CNR, Sapienza Università di Roma, 00185, Rome, Italy

${ }^{2}$ Aix-Marseille Univ, CNRS, Architecture et Fonction des Macromolécules Biologiques (AFMB), UMR 7257, 13288, Marseille, France

*Correspondence to: stefano.gianni@uniroma1.it 


\begin{abstract}
The KIX domain is an 89-residues globular domain with an important role in mediating protein-protein interactions. The presence of two distinct binding sites in such a small domain makes KIX a suitable candidate to investigate the effect of the potentially divergent demands between folding and function. Here, we present an extensive mutational analysis of the folding pathway of the KIX domain, based on 30 site-directed mutants, which allow us to provide structural information on both the transition and denatured states. Data reveal that, while the transition state resembles a distorted version of the native state, the denatured state is somewhat misfolded. We mapped some of the non-native contacts in the denatured state using a second round of mutagenesis, based on double mutant cycles on 15 double mutants. Interestingly, such a misfolding arises from non-native interactions involving the residues critical for the function of the protein. Taken together, our results indicate that the competition between folding and function creates the possibility of misfolding, which may be observed in the early stages of folding.
\end{abstract}

Keywords: Denatured state; Kinetics; Misfolding; Transition state 
A fundamental goal of protein folding studies is to describe the mechanism whereby a denatured protein achieves its native conformation. However, a major complexity arises from the co-operativity of the folding process - despite hundreds of weak noncovalent interactions between the residues and the solvent are being formed and broken, the native and denatured states are the only species significantly populated (Jackson, 1991 \#1;Jackson, 1970 \#2;Tanford, 1968 \#3). Thus, although productive folding intermediates (Capaldi, 2001 \#6;Jemth, 2004 \#7;Parker, 1995 \#8;Shastry, 1998 \#9;Ivarsson, 2007 \#10;Travaglini-Allocatelli, 2003 \#11) or off-pathway kinetic traps (Gianni, 2010 \#5;Ivarsson, 2008 \#4) may be sometimes experimentally detected, the folding reaction of small globular proteins conforms very frequently to a simple as two-state reaction.

Because of the extraordinary high degree of co-operativity intrinsic to a two-state folding mechanism, structural information about the pathway may only be inferred indirectly. In fact, systematic perturbation of the structure of the protein, by conservative site-directed mutations, allows mapping the interaction pattern present in both the transition and denatured states (Fersht, 1992 \#12;Fersht, \#13). This experimental characterization is critical to identify the formation of one or more folding nuclei, stabilizing the transition state, as well as to detect the residual structure in the denatured state, which under physiological conditions appears to play a major role in sculpting both the folding pathway and the native topology (McCarney, 2005 \#14;Morrone, 2011 \#15;Religa, 2005 \#16;van Gunsteren, 2001 \#17;Wong, 2000 \#18;Camilloni, 2016 \#19).

The KIX domain is a globular domain that is a part of a large coactivator protein 
called CBP, whose three-dimensional structure consists of three $\alpha$-helices and two short $3_{10}$-helices (Radhakrishnan, 1997 \#21;Zor, 2004 \#22). From a physiological perspective, KIX is capable of recognizing and binding, via two distinct binding sites located at opposite sides of the domain, several intrinsically unstructured proteins, which all fold upon binding (Brüschweiler, 2009 \#23;Law, 2014 \#24;Lee, 2009 \#20;Toto, 2016 \#25;Toto, 2014 \#26;Toto, 2016 \#27). These two binding sites are named after two characteristic ligands of KIX: the c-Myb site after the transactivation domain of the oncoprotein c-Myb, and the MLL site after the Mixed Lineage Leukemia protein. The folding of the KIX domain was previously analyzed by equilibrium and kinetic experiments. Whilst earlier work suggested the presence of a reaction intermediate, based on a curvature in the dependence of the logarithm of the folding rate constant from denaturant concentration (Horng, 2005 \#46), recent kinetic re-analysis of the folding of this globular domain demonstrated that this curvature was an artifact arising from time limitation of the stopped-flow apparatus (Morrone, $2012 \# 29$ ). Thus, we concluded that folding of KIX is consistent with a two-state scenario.

In this work we present the experimental characterization of the folding pathway of the KIX domain, as probed by the equilibrium and kinetic analysis of 30 site directed variants, providing structural information on both the transition and denatured states. Data reveal that, whilst the folding transition state resembles a distorted version of the native state, the denatured state of the protein appears to contain non-native interactions. Furthermore, we observe that some of the mutations located in the functional sites of KIX are associated to a stabilization of the domain, a result that appears to highlight the divergent requirements of folding and function. 


\section{RESULTS}

To map the structural features of the folding pathway of the KIX domain we performed a $\Phi$ value analysis. By following this methodology (Fersht, \#13;Fersht, $1992 \# 12$ ), the polypeptide chain is systematically mutated and the extent of the contacts formed by any given residue in the transition state is measured by the $\Phi$ value. Quantitatively, the $\Phi$ value is expressed by normalizing the change in activation free energy by the change in stability of the native state associated to the mutation. Therefore, we produced 30 site directed variants of KIX, listed in Table 1, out of which 7 did not express to a significant extent. The variants were designed by following the guidelines typically adopted in $\Phi$ value analysis. In particular, a conservative truncation of the side chain was introduced in each hydrophobic position of the domain; a type of mutation that, as extensively discussed previously(Fersht, \#13;Fersht, 1992 \#12), represents the most easy to interpret in this kind of analysis. All the purified variants were subjected to equilibrium and kinetic folding experiments.

Urea-induced equilibrium denaturation of KIX and its site directed variants was monitored by intrinsic Trp emission at $20{ }^{\circ} \mathrm{C}$, pH 7.2 in $50 \mathrm{mM}$ sodium phosphate buffer in the presence of $150 \mathrm{mM} \mathrm{NaCl}$. In all cases, the observed transitions followed a simple two-state behavior (Figure 1), suggesting the absence of stable equilibrium intermediate(s). The thermodynamic stability and apparent $m_{\mathrm{D}-\mathrm{N}}$ value for each mutant, derived from two-state analysis described below, are reported in Table 1.

Unfolding and refolding kinetics of all the proteins were measured by stopped-flow 
kinetics, by 11-fold dilution of the denatured or the native protein into solutions containing different urea concentrations. In all experiments, the fluorescence time course was satisfactorily fitted to a single-exponential decay. The semilogarithmic plots of the observed unfolding and refolding rate constants versus denaturant concentration (chevron plots) for wild-type KIX and all the variants are shown in Figure 1. In agreement with our previous observations for wild type KIX (Morrone, 2012 \#29), all site directed mutants displayed a V-shaped chevron plot and none of them displayed any deviation from linearity in the folding and unfolding limbs. This finding reinforces our previous proposal for a two-state mechanism of this globular domain and the absence of detectable transient intermediates; a model appearing in this case also rather robust with respect to site-directed mutagenesis.

Because of its small size (89 amino acids) (Myers, 1995 \#30), KIX displays a relatively low cooperativity, the $m_{\mathrm{D}-\mathrm{N}}$ obtained from equilibrium urea-induced denaturation being $0.95+/-0.08 \mathrm{kcal} \mathrm{mol}^{-1} \mathrm{M}^{-1}$; this complicates an accurate determination of the folding parameters, from equilibrium experiments alone. Thus, the equilibrium and kinetic experiments of each variant were fitted globally to the following equations:

Equilibrium: $Y_{o b s}=Y_{N}+Y_{D} \frac{e^{\left(m_{D-N}\left([\text { urea }][\text { urea }]_{1 / 2}\right)\right.}}{1+e^{\left(m_{D-N}\left([\text { urea }]-[\text { urea }]_{1 / 2}\right)\right.}}$

Kinetics: $\quad k_{o b s}=k_{F} e^{\left(-m_{F}[\text { urea }]\right)}+k_{U} e^{\left(-m_{U}[\text { urea }]\right)}$

In each case, the $m_{\mathrm{D}-\mathrm{N}}$ value (equivalent to the sum between $m_{\mathrm{F}}$ and $m_{\mathrm{U}}$ ) was shared between the equilibrium and kinetic data sets, in agreement with the two-state 
formalism (Jackson, 1991 \#1). This fitting procedure allowed (i) to test the robustness of the two-state mechanism for each individual mutant and (ii) to decrease the fitting error on $m_{\mathrm{D}-\mathrm{N}}$ arising from the low cooperativity observed in the equilibrium transition.

Following a generally accepted convention (Fersht, 2004 \#31;Fersht, 1995 \#32), the experimentally determined $\Phi$ values were divided in three different groups and mapped onto the native structure of KIX (Figure 2A): small values $(\Phi<0.3$; red), intermediate values $(0.3<\Phi<0.7$; magenta $)$, and large values $(\Phi>0.7$; blue $)$. The structural distribution of the $\Phi$ values indicates that the transition state has a rather diffuse native-like structure with a folding nucleus approximately located by and large in the C-terminal helix. To address the overall structural features of the transition state we performed a linear free energy relationship analysis (LFER) (Leffler, 1953 \#33). By following this method, the changes in free energy of the transition state are related to changes in equilibrium free energies, with the slope of the observed correlation (called $\alpha$ value), reflecting the degree of similarity between the native and transition states. As depicted in Figure 2B, in the case of KIX the LFER yields a linear correlation with a slope of 0.67 . This is consistent with what observed in other globular proteins and indicates that KIX folds via the so called nucleationcondensation mechanism (Fersht, 1995 \#35;Itzhaki, 1995 \#36). This description implies that the transition state resembles a distorted version of the native state and the whole protein self-assembles around a weakly formed nucleus.

A reliable method to investigate the global properties of folding transition and denatured states is the analysis of chevron plots recorded under different experimental 
conditions or on various site-directed variants (Sanchez, 2003 \#37;Scaloni, 2009 \#38). In fact, slopes of the unfolding and refolding arms of the chevron plot (yielding the $m_{\mathrm{U}}$ and $m_{\mathrm{F}}$ values respectively) reflect the change in accessible surface area upon (un)folding; an analysis of their dependence on reaction conditions may be of diagnostic value to detect shifts of the transition and denatured states along the reaction coordinate. The dependence of the $m_{\mathrm{D}-\mathrm{N}}, m_{\mathrm{U}}$ and $m_{\mathrm{F}}$ versus the change in free energy upon mutation is reported in Figure 3. Interestingly, it is clear that, whilst $m_{\mathrm{U}}$ is essentially insensitive to the thermodynamic stability of KIX, both the $m_{\mathrm{F}}$ and $m_{\mathrm{D}-\mathrm{N}}$ display a similar linear dependence and decrease with decreasing protein stability. Since $m_{\mathrm{U}}$ does not change with overall thermodynamic stability, these observations indicate that the changes in the folding $m$ values are due to movements of the denatured state towards the native state (a compaction) and not by movements of the transition state. Thus, the experiments detect a residual malleable structure in the denatured state of KIX, which is perturbed by mutagenesis. Interestingly, this compaction of the denatured state upon thermodynamic destabilization indicates that, contrary to what has been previously observed in other proteins, the residual structure is largely due to non-native interactions.

Investigating non-native interactions in the denatured state of KIX by double mutant cycles.

The changes in free energy of folding introduced by site directed mutagenesis depend on the effect of a given mutation on both the denatured and native states. In particular, the experimentally measured $\Delta \Delta \mathrm{G}_{\mathrm{D}-\mathrm{N}}=\Delta \Delta \mathrm{G}_{\mathrm{N}-\mathrm{N}},-\Delta \Delta \mathrm{G}_{\mathrm{D}-\mathrm{D}}$, where $\mathrm{D}^{\prime}$ and $\mathrm{N}^{\prime}$ represent the denatured and native states of the mutated protein respectively. Because residues are generally assumed not to interact in unfolded conformations, the observed $\Delta \Delta \mathrm{G}_{\mathrm{D}-\mathrm{N}}$ 
typically equals $\Delta \Delta \mathrm{G}_{\mathrm{N-N}} ;$ hence, in the absence of residual structure in the denatured state, the observed change in stability reflects the perturbation in the free energy of the native state induced by the mutation.

Analogously, by considering a cubic thermodynamic scheme, the sum of the changes in stability introduced by two single mutants can be compared with the corresponding double mutant to measure a coupling free energy $\Delta \Delta \Delta \mathrm{G}_{\mathrm{D}-\mathrm{N}}=\Delta \Delta \Delta \mathrm{G}_{\mathrm{N}-\mathrm{N}},-\Delta \Delta \Delta \mathrm{G}_{\mathrm{D}^{-\mathrm{D}}{ }^{\prime}}$ where $\Delta \Delta \Delta \mathrm{G}_{\mathrm{N}-\mathrm{N}}$, and $\Delta \Delta \Delta \mathrm{G}_{\mathrm{D}-\mathrm{D}}$, represent a measure of the strength with which the two residues interact in the native and denatured states respectively. A $\Delta \Delta \Delta \mathrm{G}_{\mathrm{D}-\mathrm{N}}$ tending to 0 would be observed when the effect of the double mutant is similar to the sum of the two single mutants, whereas a $\Delta \Delta \Delta \mathrm{G}_{\mathrm{D}-\mathrm{N}} \neq 0$ implies that the probed positions interact energetically.

To provide additional evidence for the presence of non-native interactions in the denatured state of KIX, we resorted to perform double mutant cycles. In particular, we focused on those positions displaying an increase in $m_{\mathrm{D}-\mathrm{N}}$ value of more than 0.1 kcal mol ${ }^{-1} \mathrm{M}^{-1}$ (i.e. the mutations that present a detectable increase of the accessible surface area of the denatured state) and produced the different double mutants. Six mutations were therefore taken into account (L18A, I26V, L43A, Y65A, L67A and Y73) and the 15 corresponding double mutants were produced. Twelve of them were stable enough to be expressed, purified and characterized. In analogy to what described above, the 12 double mutants were subjected to equilibrium and kinetic experiments (Figure 4) and the calculated folding parameters are reported in Table 2, together with their associated $\Delta \Delta \Delta \mathrm{G}_{\mathrm{D} \text {-TS }}$ and $\Delta \Delta \Delta \mathrm{G}_{\mathrm{D}-\mathrm{N}}$. These values were obtained from the difference of the effect observed for the double mutant minus the individual contribution of the each of the two single variants. 
Inspection of Table 2 reveals that, in 6 cases, there was a detectable coupling free energy $\Delta \Delta \Delta \mathrm{G}_{\mathrm{D}-\mathrm{N}}$ between couples of residues. Remarkably, with the exception of positions I26 and L43 that are in direct contact in the native state of KIX, all the other pairs of residues displaying a $\Delta \Delta \Delta \mathrm{G}_{\mathrm{D}-\mathrm{N}}$ are located in different regions of the domain, suggesting the value of $\Delta \Delta \Delta \mathrm{G}_{\mathrm{N}-\mathrm{N}} \approx 0$. In those cases, the $\Delta \Delta \Delta \mathrm{G}_{\mathrm{D}-\mathrm{N}}$ would tend to the value of $\Delta \Delta \Delta \mathrm{G}_{\mathrm{D}-\mathrm{D}}$, and would be therefore diagnostic of a non-native contact in the denatured state. In support of this view, it is of interest to comment of the magnitude of coupling free energies in the folding transition state, which appear to provide an internal control. In fact, by considering the equation $\Delta \Delta \Delta \mathrm{G}_{\mathrm{D}-\mathrm{TS}}=\Delta \Delta \Delta \mathrm{G}_{\mathrm{TS}-\mathrm{TS}}$ ' $\underline{\Delta \Delta \Delta \mathrm{G}_{\mathrm{D}-\mathrm{D}} \text {, }}$, since both the $\Phi$ value and LFER analysis suggests the transition state to be native-like, if the coupling free energy $\Delta \Delta \Delta \mathrm{G}_{\mathrm{D}-\mathrm{N}}$ is mainly represented by the $\underline{\Delta \Delta \Delta \mathrm{G}_{\mathrm{D}-\mathrm{D}} \text {, }}$ it follows that, in the absence of non-native like interactions in TS,

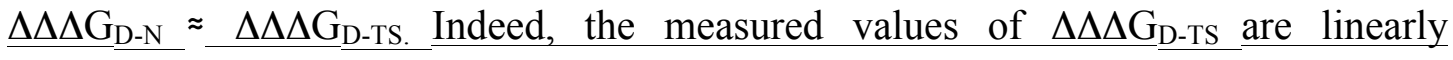
correlated with the values of $\Delta \Delta \Delta \mathrm{G}_{\mathrm{D}-\mathrm{N}}$ (Table 2), displaying a slope of 0.93 and and $\underline{\mathrm{R}^{2}=0.87 \text { (not shown), confirming that the coupling free energies measured by double }}$ mutant cycles report mainly on effects on the denatured state.

\section{DISCUSSION}

The early work in enzymology has shown that catalysis is improved when reaction intermediates tend to be minimally populated (Fersht, \#13;Pauling, 1946 \#39). Similarly, in the case of folding, it has been proposed that optimization of folding rate constants, which might be vital for productive folding, may be achieved from the concurrent formation of secondary and tertiary structure from a highly disordered 
denatured state (Fersht, 1995 \#35), i.e. by following the so-called nucleationcondensation mechanism. Consequently, it has been proposed that there is an evolutionary pressure contrasting the formation of stable structural elements in denatured states.

In this work, we have extensively characterized the folding of the KIX domain by studying the equilibrium and kinetic properties of a large number of site-directed variants, in comparison to that of the wild-type protein. Whilst the transition state displays the classical signatures of the nucleation condensation-mechanism, with a native-like topology and a linear LFER plot (Figure 2), analysis of the denatured state properties reveals an additional complexity. In fact, the folding and unfolding $m$ values of this globular domain allows detecting the presence of a residual structure in the denatured state, which appears to be mainly stabilized by non-native interactions. We mapped some of these non-native interactions by performing double mutant cycles on a limited set of variants displaying an experimentally detectable increase of $\underline{m_{D-N}} \underline{\text { value (i.e. those variants which significantly perturbed the residual structure in }}$ the denatured state by increasing its accessible surface area).

The energy landscape theory proposes that proteins, while marginally stable, are strongly biased towards their native structure, which is therefore minimally frustrated (Bryngelson, $1995 \# 40$ ). Because of the different demands of folding and function, however, it is plausible to assume that the functionally competent site(s) of a protein may contain a certain level of local frustration, such that, in those regions, the sequence is not necessarily optimized to fold (Ferreiro, 2007 \#41;Ferreiro, 2011 \#42). The presence of these patterns may lead to local misfolding, which may be 
observed in the early events of folding (Di Silvio, 2015 \#43;Gianni, 2014 \#44). By following this hypothesis, it is of interest to localize on the three dimensional structure of KIX the sites of mutations that result in a relevant increase of $m_{\mathrm{D}-\mathrm{N}}$ value (i.e. the mutants whose $m$-value is at least $0.1 \mathrm{kcal} \mathrm{mol}^{-1} \mathrm{M}^{-1}$ higher than the wild type; Figure 5). In fact, because variations at these positions yield to an expansion of the denatured chain, it is likely that they are directly involved in the formation of nonnative interactions. Consistently, inspection of Figure 5 suggests that the positions directly involved in residual structure of the denatured state are indeed located in the binding sites of the protein. Three of these mutations, also highlighted in Figure 5, resulted in a detectable stabilization of the native state, providing further support on the hypothesis that sequence is not optimized to fold in these regions. A more direct, even if limited, investigation of the presence of non-native interaction patterns in the denatured state of KIX is represented by the presence of a detectable coupling free energy $\Delta \Delta \Delta \mathrm{G}_{\mathrm{D}-\mathrm{N}}$ between residues not directly interacting in the native state. Within these residues (mapped on the structure of KIX in Figure 5C), it appears that position Y73, located in $\alpha 3$ of KIX located in the c-Myb binding pocket, plays a particularly important role in stabilizing the residual structure of the denatured state. This finding parallels the earlier NMR observations by Tollinger and co-workers (Schanda, 2008 \#47;Tollinger, 2006 \#48), who highlighted the capability of $\alpha 3$ to fold independently from the rest of the domain.

Because of its functional complexity, the KIX domain is an interesting system for protein folding studies. In fact, despite its small size and simple topology, KIX interacts with different proteins via two distinct binding sites, which occupy a large portion in the surface of the domain. In this perspective, the experimental results 
described in this work offer the opportunity to test the effects of the divergent demands of folding and function in proteins. The structural distribution of the residues involved in non-native interactions in the denatured state of KIX appears to confirm the notion that the misfolding that may occur in the early events of folding arises from the residues involved in the function of the protein.

\section{MATERIALS AND METHODS}

\section{Site-Directed Mutagenesis and Protein Expression and Purification.}

KIX wild-type and all the site directed mutants was expressed and purified as described previously (Giri, 2013 \#45;Morrone, 2012 \#29). All the site-directed mutants were obtained by using the QuikChange mutagenesis kit (Stratagene) according to the manufacturer's instructions. Mutations were confirmed by DNA sequencing. The purity of the protein was confirmed by SDS-PAGE.

\section{Stopped-flow measurements.}

The unfolding and refolding kinetics experiments were carried out on a single-mixing SX-18 stopped-flow instrument (Applied Photophysics, Leatherhead, UK); the excitation wavelength was $280 \mathrm{~nm}$ and the fluorescence emission was collected using a $320 \mathrm{~nm}$ cut-off glass filter. The experiments on KIX wild-type and its variants were performed at $20^{\circ} \mathrm{C}$ by mixing 11 -fold dilution of the denatured or the native protein in $50 \mathrm{mM}$ sodium phosphate and $150 \mathrm{mM} \mathrm{NaCl}, \mathrm{pH} 7.2$, into solutions containing different urea concentrations. Final protein concentration was typically $3 \mu \mathrm{M}$.

\section{Equilibrium experiments.}


Equilibrium unfolding experiments on KIX wild-type and its variants, were carried on a Fluoromax single photon counting spectrofluorometer (Jobin-Yvon, NJ, USA), by mixing the native protein with increasing urea concentrations at $20{ }^{\circ} \mathrm{C}$, in $50 \mathrm{mM}$ sodium phosphate and $150 \mathrm{mM} \mathrm{NaCl}, \mathrm{pH} \mathrm{7.2,} \mathrm{measuring} \mathrm{the} \mathrm{intrinsic} \mathrm{tryptophan}$ emission. Tryptophan fluorescence emission spectra were recorded in a cuvette $(1 \mathrm{~cm}$ light path) between 300 and $400 \mathrm{~nm}$. The excitation wavelength was $280 \mathrm{~nm}$. Proteins concentration was typically $2 \mu \mathrm{M}$.

\section{ACKNOWLEDGEMENTS}

Work partly supported by grants from the Italian Ministero dell'Istruzione dell'Università e della Ricerca (Progetto di Interesse 'Invecchiamento' to S.G.) and Sapienza University of Rome (C26A155S48 to S.G).

\section{REFERENCES}




\section{FIGURE LEGENDS.}

Figure 1. Equilibrium denaturations and chevron plots of KIX and its site directed mutants. All the experiments were carried out at $20{ }^{\circ} \mathrm{C}, \mathrm{pH} 7.2$ in $50 \mathrm{mM}$ sodium phosphate buffer in the presence of $150 \mathrm{mM} \mathrm{NaCl}$. As described in the Results section, each mutant was globally fitted to a two state mechanism by assuming the $m_{\mathrm{D}-\mathrm{N}}$ value at equilibrium to be equivalent to the sum between the kinetic $m_{\mathrm{F}}$ and $m_{\mathrm{U}}$ values (Jackson, 1991 \#1)

Figure 2. The folding transition state of the KIX domain. Panel A. Following a generally accepted convention (Fersht, 2004 \#31;Fersht, 1995 \#32), the experimentally determined $\Phi$ values were divided in three different groups and mapped onto the native structure of KIX: small values $(\Phi<0.3$; red), intermediate values $(0.3<\Phi<0.7$; magenta), and large values $(\Phi>0.7$; blue). Panel B. LFER analysis of the transition state of KIX. In agreement with the nucleation condensation mechanism (Fersht, 1995 \#35), we observed a linear LFER plot with an apparent $\alpha$ value of $0.67 \pm 0.09$

Figure 3. Dependence of $m_{\mathrm{F}}$ (squares), $m_{\mathrm{U}}$ (open circles) and $m_{\mathrm{D}-\mathrm{N}}($ filled) on protein stability. Lines are the best fit to a linear function. As discussed in the text, both $m_{\mathrm{F}}$ and $m_{\mathrm{D}-\mathrm{N}}$ increase in parallel with increasing protein stability, strongly indicating that KIX displays residual malleable structure in its denatured state, which is primarily stabilized by non-native interactions. 
Figure 4. Double mutant cycles in the KIX domain. All the experiments were carried out at $20{ }^{\circ} \mathrm{C}, \mathrm{pH} 7.2$ in $50 \mathrm{mM}$ sodium phosphate buffer in the presence of $150 \mathrm{mM}$ $\mathrm{NaCl}$. As described in the Results section, each mutant was globally fitted to a two state mechanism by assuming the $m_{\mathrm{D}-\mathrm{N}}$ value at equilibrium to be equivalent to the sum between the kinetic $m_{\mathrm{F}}$ and $m_{\mathrm{U}}$ values (Jackson, 1991 \#1)

Figure 5. A. Structural distribution of variants with increased $m_{\mathrm{D}-\mathrm{N}}$ in the KIX domain. The variants of KIX displaying a detectable increase of $m_{\mathrm{D}-\mathrm{N}}$ value (i.e. the mutants whose $m$-value is at least $0.1 \mathrm{kcal} \mathrm{mol}^{-1} \mathrm{M}^{-1}$ higher than the wild type) are mapped onto the structure and represented in sphere. As discussed in the text, it is evident that all the variants are located in the binding sites for either c-Myb (represented in yellow) or MLL (represented in black). The three variants resulting in a relevant stabilization of the native state (i.e. L18A, L43A and Y73A) are colored in salmon. B. Structural distribution of the residues displaying a role in stabilizing nonnative interactions in the denatured state of KIX as probed by double mutant cycles. 
Table 1. Folding parameters for KIX and its site-directed variants measured from equilibrium and kinetic experiments.

\begin{tabular}{|l|c|c|c|c|c|c|c|}
\hline & $\begin{array}{c}k_{\mathrm{F}} \\
\left(\mathrm{s}^{-1}\right)\end{array}$ & $\begin{array}{c}k_{U} \\
\left(\mathrm{~s}^{-1}\right)\end{array}$ & $\begin{array}{c}\mathrm{m}_{\mathrm{F}} \\
(\mathrm{kcal} / \mathrm{M} \mathrm{mol})\end{array}$ & $\begin{array}{c}\mathrm{m}_{U} \\
(\mathrm{kcal} / \mathrm{M} \mathrm{mol})\end{array}$ & $\begin{array}{c}\mathrm{m}_{\mathrm{D}-\mathrm{N}} \\
(\mathrm{kcal} / \mathrm{M} \mathrm{mol})\end{array}$ & $\begin{array}{c}\Delta \Delta \mathrm{G}_{\mathrm{D}-\mathrm{N}} \\
(\mathrm{kcal} / \mathrm{mol})\end{array}$ & $\phi$ \\
\hline $\mathrm{WT}$ & $2400 \pm 300$ & $14.1 \pm 0.9$ & $0.76 \pm 0.04$ & $0.20 \pm 0.06$ & $0.96 \pm 0.04$ & - & - \\
\hline T11S & $2100 \pm 200$ & $21 \pm 2$ & $0.76 \pm 0.08$ & $0.2 \pm 0.1$ & $0.92 \pm 0.09$ & $0.29 \pm 0.11$ & $*$ \\
\hline L14A & $1700 \pm 200$ & $11 \pm 1$ & $0.73 \pm 0.07$ & $0.23 \pm 0.09$ & $0.96 \pm 0.05$ & $0.05 \pm 0.11$ & $*$ \\
\hline L18A & $1700 \pm 300$ & $3.0 \pm 0.3$ & $0.86 \pm 0.03$ & $0.20 \pm 0.04$ & $1.06 \pm 0.03$ & $-0.71 \pm 0.13$ & $-0.26 \pm 0.17$ \\
\hline V23A & $720 \pm 70$ & $35 \pm 3$ & $0.51 \pm 0.05$ & $0.17 \pm 0.09$ & $0.68 \pm 0.07$ & $1.21 \pm 0.11$ & $0.57 \pm 0.09$ \\
\hline A25G & $740 \pm 70$ & $84 \pm 8$ & $0.46 \pm 0.05$ & $0.2 \pm 0.1$ & $0.60 \pm 0.09$ & $1.72 \pm 0.11$ & $0.39 \pm 0.06$ \\
\hline I26V & $2400 \pm 200$ & $23 \pm 2$ & $0.93 \pm 0.02$ & $0.18 \pm 0.02$ & $1.1 \pm 0.1$ & $0.29 \pm 0.11$ & $*$ \\
\hline T29S & $1400 \pm 100$ & $11 \pm 1$ & $0.65 \pm 0.06$ & $0.2 \pm 0.1$ & $0.86 \pm 0.08$ & $0.20 \pm 0.11$ & $*$ \\
\hline A33G & $3100 \pm 500$ & $21 \pm 3$ & $0.84 \pm 0.04$ & $0.15 \pm 0.06$ & $0.99 \pm 0.04$ & $0.07 \pm 0.14$ & $*$ \\
\hline A34G & $3000 \pm 300$ & $25 \pm 2$ & $0.82 \pm 0.08$ & $0.2 \pm 0.1$ & $0.99 \pm 0.06$ & $0.19 \pm 0.11$ & $*$ \\
\hline L35A & $2600 \pm 300$ & $25 \pm 2$ & $0.82 \pm 0.08$ & $0.2 \pm 0.1$ & $0.99 \pm 0.06$ & $0.29 \pm 0.11$ & $*$ \\
\hline L43A & $2100 \pm 300$ & $1.3 \pm 0.2$ & $0.90 \pm 0.03$ & $0.26 \pm 0.04$ & $1.16 \pm 0.04$ & $-1.31 \pm 0.14$ & $-0.05 \pm 0.08$ \\
\hline V44A & $630 \pm 70$ & $70 \pm 8$ & $0.62 \pm 0.07$ & $0.14 \pm 0.09$ & $0.76 \pm 0.06$ & $1.70 \pm 0.12$ & $0.45 \pm 0.06$ \\
\hline A45G & $1300 \pm 100$ & $27 \pm 2$ & $0.82 \pm 0.03$ & $0.17 \pm 0.04$ & $0.99 \pm 0.03$ & $0.73 \pm 0.10$ & $0.47 \pm 0.13$ \\
\hline Y46A & $1800 \pm 400$ & $63 \pm 9$ & $0.76 \pm 0.09$ & $0.2 \pm 0.1$ & $0.9 \pm 0.1$ & $1.03 \pm 0.17$ & $0.15 \pm 0.14$ \\
\hline V50A & $1900 \pm 200$ & $35 \pm 3$ & $0.62 \pm 0.06$ & $0.18 \pm 0.08$ & $0.80 \pm 0.05$ & $0.65 \pm 0.11$ & $0.20 \pm 0.14$ \\
\hline Y65A & $1800 \pm 200$ & $26 \pm 3$ & $1.0 \pm 0.2$ & $0.2 \pm 0.3$ & $1.2 \pm 0.2$ & $0.60 \pm 0.12$ & $0.43 \pm 0.17$ \\
\hline L67A & $1600 \pm 300$ & $30 \pm 5$ & $0.9 \pm 0.1$ & $0.2 \pm 0.2$ & $1.1 \pm 0.1$ & $0.50 \pm 0.18$ & $-0.03 \pm 0.29$ \\
\hline L68A & $260 \pm 30$ & $7 \pm 1$ & $0.6 \pm 0.1$ & $0.26 \pm 0.04$ & $0.9 \pm 0.1$ & $0.90 \pm 0.12$ & $1.43 \pm 0.22$ \\
\hline A69G & $1200 \pm 100$ & $13 \pm 1$ & $0.79 \pm 0.08$ & $0.22 \pm 0.09$ & $1.01 \pm 0.04$ & $0.35 \pm 0.11$ & $*$ \\
\hline I72V & $2400 \pm 400$ & $24 \pm 3$ & $0.85 \pm 0.06$ & $0.18 \pm 0.08$ & $1.03 \pm 0.06$ & $0.29 \pm 0.15$ & $*$ \\
\hline Y73A & $6000 \pm 1000$ & $15 \pm 3$ & $0.89 \pm 0.07$ & $0.22 \pm 0.09$ & $1.11 \pm 0.05$ & $-0.53 \pm 0.17$ & $1.00 \pm 0.41$ \\
\hline I75V & $2300 \pm 400$ & $24 \pm 4$ & $0.74 \pm 0.05$ & $0.15 \pm 0.06$ & $0.89 \pm 0.04$ & $0.32 \pm 0.16$ & $*$ \\
\hline L79A & $2100 \pm 200$ & $8 \pm 1$ & $0.81 \pm 0.03$ & $0.21 \pm 0.04$ & $1.02 \pm 0.02$ & $-0.24 \pm 0.13$ & $*$ \\
\hline
\end{tabular}

The mutants V10A, V19A, L22A, A47G, Y55A, A58G and Y64A expressed poorly and could not be characterized.

* These mutants show $\Delta \Delta \mathrm{G}_{\mathrm{D}-\mathrm{N}}<0.40 \mathrm{kcal} / \mathrm{mol}$, preventing reliable calculation of the $\Phi$-values. 
Table 2. Folding parameters for the double mutant cycles in the KIX domain measured from equilibrium and kinetic experiments.

\begin{tabular}{|l|c|c|c|c|c|c|}
\hline MUTANTS & $\begin{array}{c}k_{\mathrm{F}} \\
\left(\mathrm{s}^{-1}\right)\end{array}$ & $\begin{array}{c}k_{\mathrm{U}} \\
\left(\mathrm{s}^{-1}\right)\end{array}$ & $\begin{array}{c}\Delta \Delta \mathrm{G}_{\mathrm{D}-\mathrm{N}} \\
(\mathrm{kcal} / \mathrm{mol})\end{array}$ & $\begin{array}{c}\Delta \Delta \mathrm{G}_{\mathrm{D}-\mathrm{TS}} \\
(\mathrm{kcal} / \mathrm{mol})\end{array}$ & $\begin{array}{c}\Delta \Delta \Delta \mathrm{G}_{\mathrm{D}-\mathrm{N}} \\
(\mathrm{kcal} / \mathrm{mol})\end{array}$ & $\begin{array}{c}\Delta \Delta \Delta \mathrm{G}_{\mathrm{D}-\mathrm{TS}} \\
(\mathrm{kcal} / \mathrm{mol})\end{array}$ \\
\hline WT & $2400 \pm 300$ & $14.1 \pm 0.9$ & & & & \\
\hline L18A I26V & $670 \pm 70$ & $3.6 \pm 0.6$ & $-0,07 \pm 0.13$ & $0.73 \pm 0.09$ & $-0.35 \pm 0.22$ & $-0.55 \pm 0.17$ \\
\hline L18A L43A & $380 \pm 40$ & $0.26 \pm 0.03$ & $-1,3 \pm 0.12$ & $1.06 \pm 0.09$ & $-0.76 \pm 0.23$ & $-0.81 \pm 0.34$ \\
\hline L18A Y65A & $830 \pm 90$ & $4.7 \pm 0.6$ & $-0,04 \pm 0,12$ & $0.6 \pm 0.09$ & $-0.17 \pm 0.22$ & $-0.21 \pm 0.17$ \\
\hline L18A L67A & $930 \pm 90$ & $8.3 \pm 0.8$ & $0,23 \pm 0.11$ & $0.54 \pm 0.09$ & $-0.28 \pm 0.25$ & $-0.14 \pm 0.21$ \\
\hline L18A Y73A & $2300 \pm 400$ & $1.7 \pm 0.5$ & $-1,20 \pm 0.20$ & $0.02 \pm 0.11$ & $-0.04 \pm 0.30$ & $-0.40 \pm 0.21$ \\
\hline I26V L43A & $650 \pm 60$ & $2.3 \pm 0.2$ & $-0,29 \pm 0.11$ & $0.75 \pm 0.09$ & $0.73 \pm 0.21$ & $0.68 \pm 0.16$ \\
\hline I26V L67A & $1300 \pm 300$ & $38.0 \pm 5.0$ & $0.93 \pm 0.17$ & $0.36 \pm 0.15$ & $0.02 \pm 0.28$ & $-0.14 \pm 0.23$ \\
\hline I26V Y73A & $4300 \pm 400$ & $19.0 \pm 3.0$ & $-0.16 \pm 0.12$ & $-0.35 \pm 0.09$ & $-0.09 \pm 0.24$ & $-0.22 \pm 0.18$ \\
\hline L43A L67A & $660 \pm 100$ & $1.4 \pm 0.3$ & $-0.6 \pm 0.18$ & $0.74 \pm 0.11$ & $-0.04 \pm 0.29$ & $-0.45 \pm 0.21$ \\
\hline L43A Y73A & $980 \pm 100$ & $1.10 \pm 0.1$ & $-0.97 \pm 0.11$ & $0.51 \pm 0.09$ & $-0.88 \pm 0.25$ & $-1.01 \pm 0.19$ \\
\hline Y65A Y73A & $1900 \pm 600$ & $31.0 \pm 10$ & $0.57 \pm 0.29$ & $0.11 \pm 0.19$ & $-0.61 \pm 0.36$ & $-0.53 \pm 0.25$ \\
\hline L67A Y73A & $1700 \pm 200$ & $26.0 \pm 3$ & $0.54 \pm 0.11$ & $0.19 \pm 0.09$ & $-0.42 \pm 0.28$ & $-0.54 \pm 0.21$ \\
\hline
\end{tabular}

The mutants I26V Y65A, L43A Y65A, Y65 L67A expressed poorly and could not be characterized. 

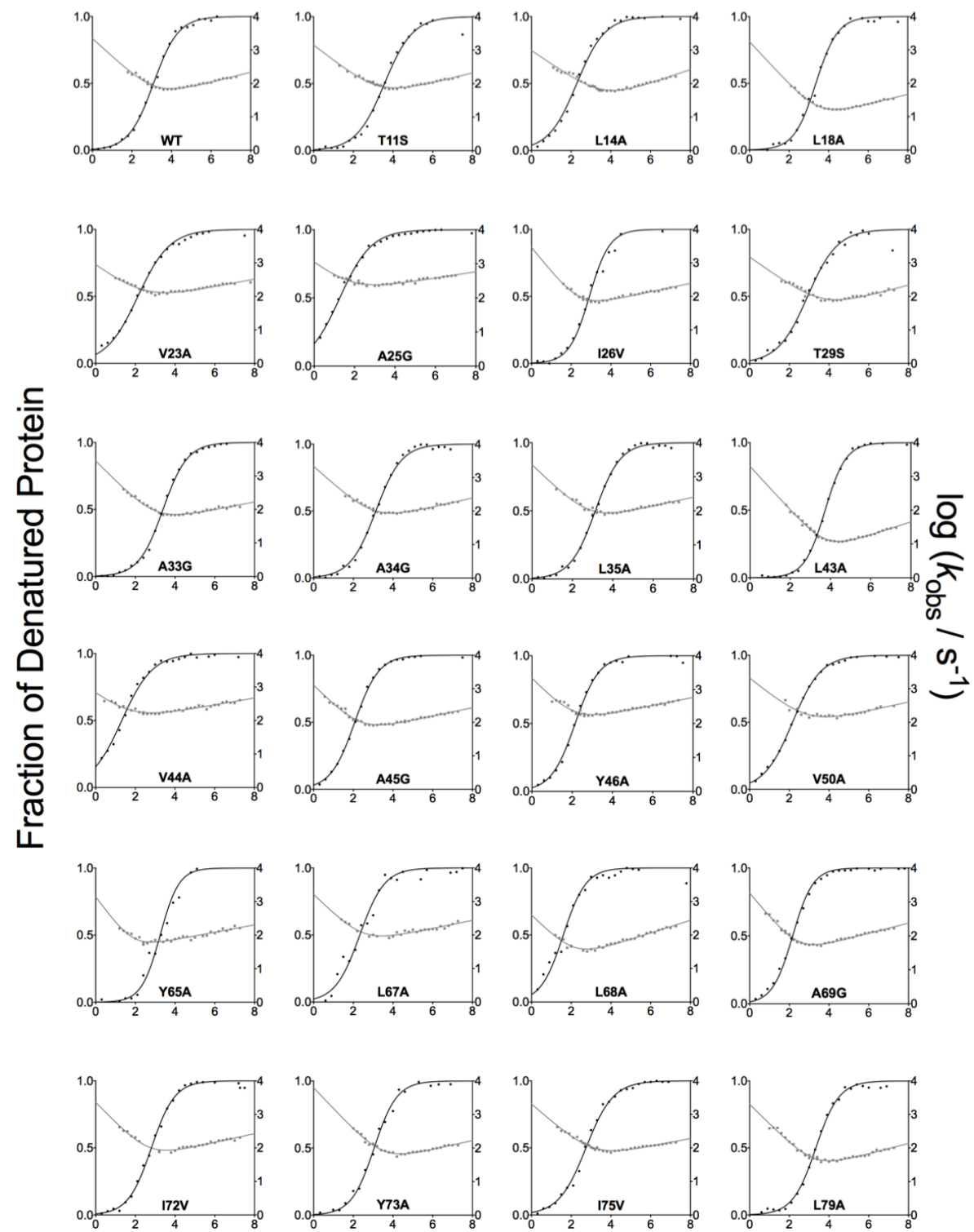

[Urea] (M)

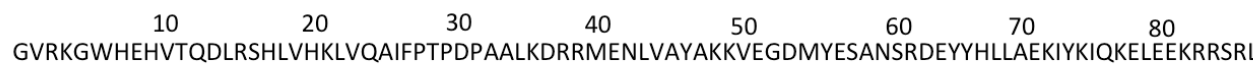

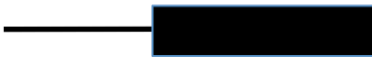

$\alpha 1$

$\alpha 2$

Figure 1 

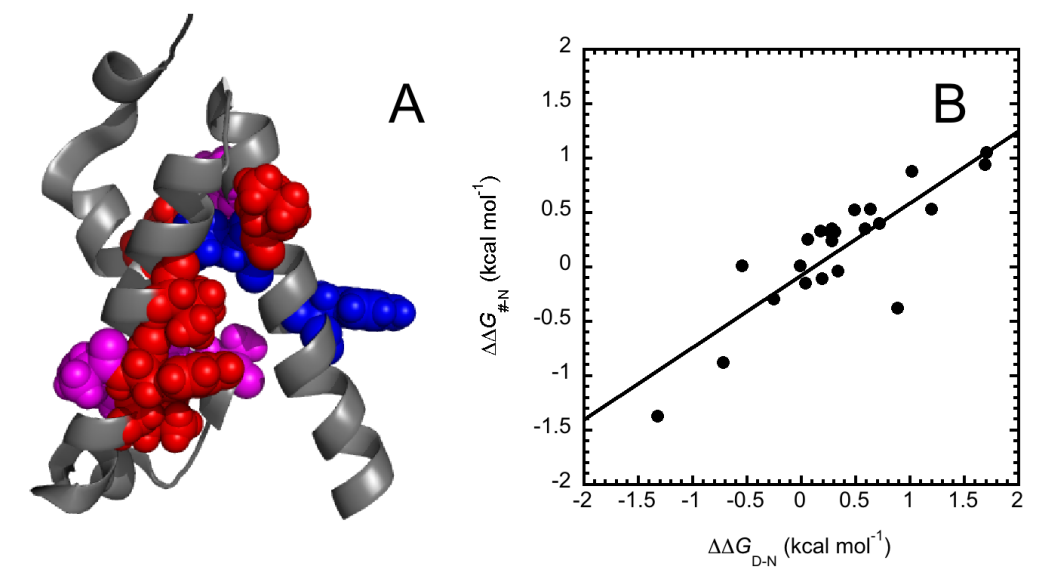

Figure 2 


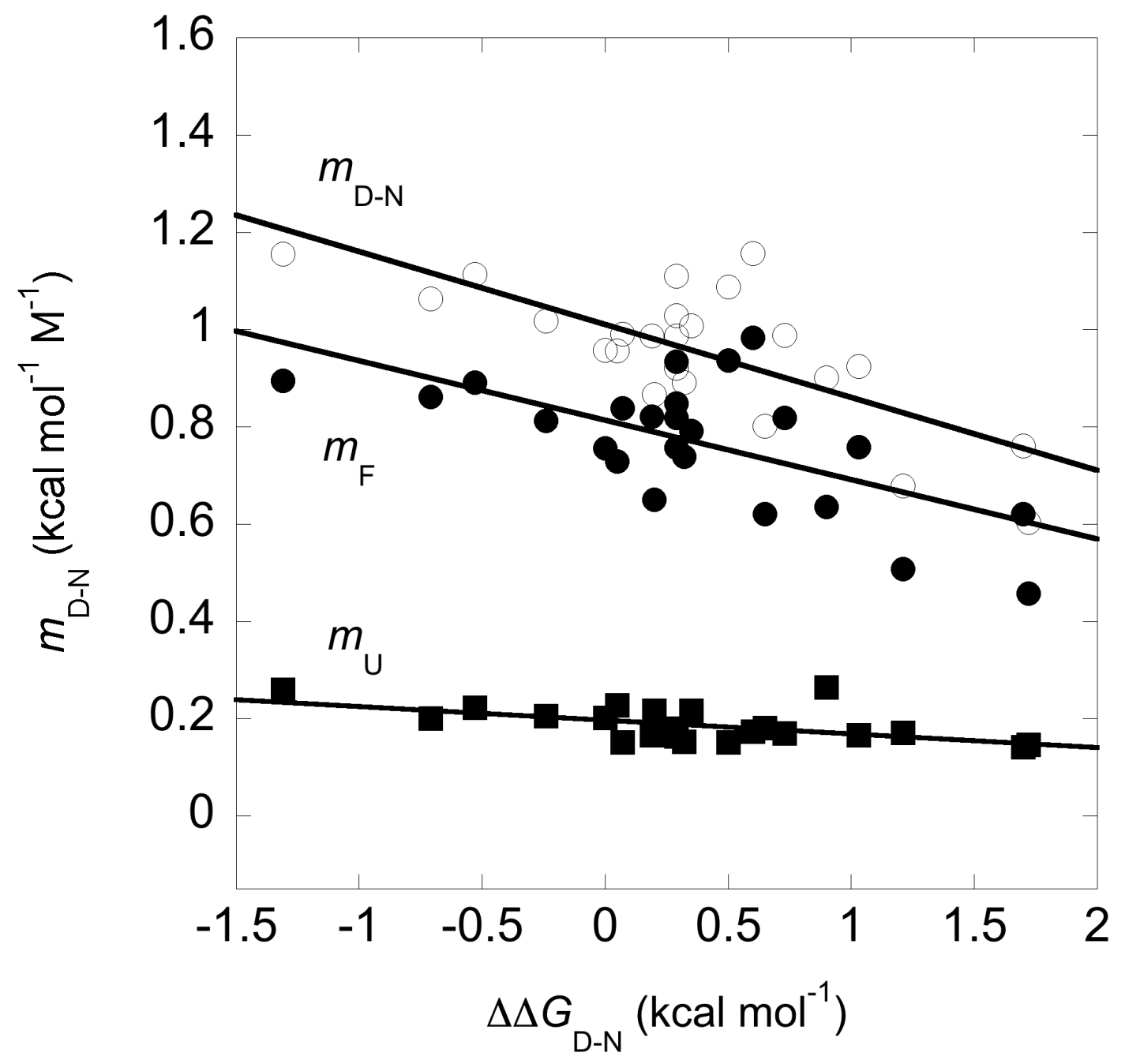

Figure 3 

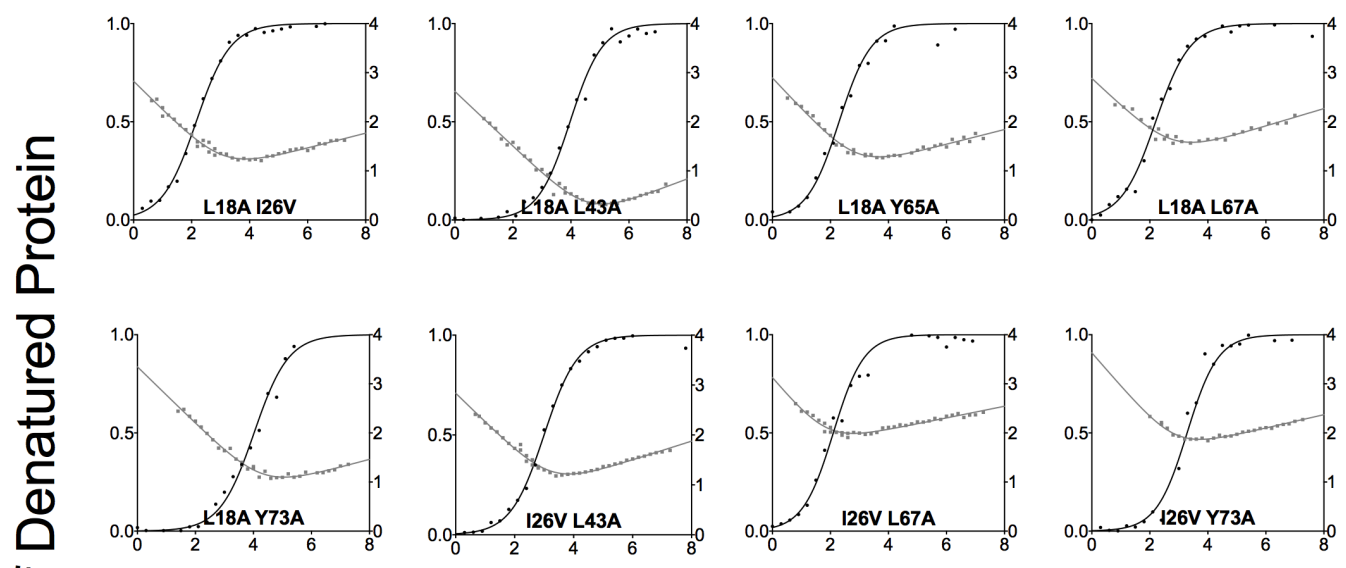

는
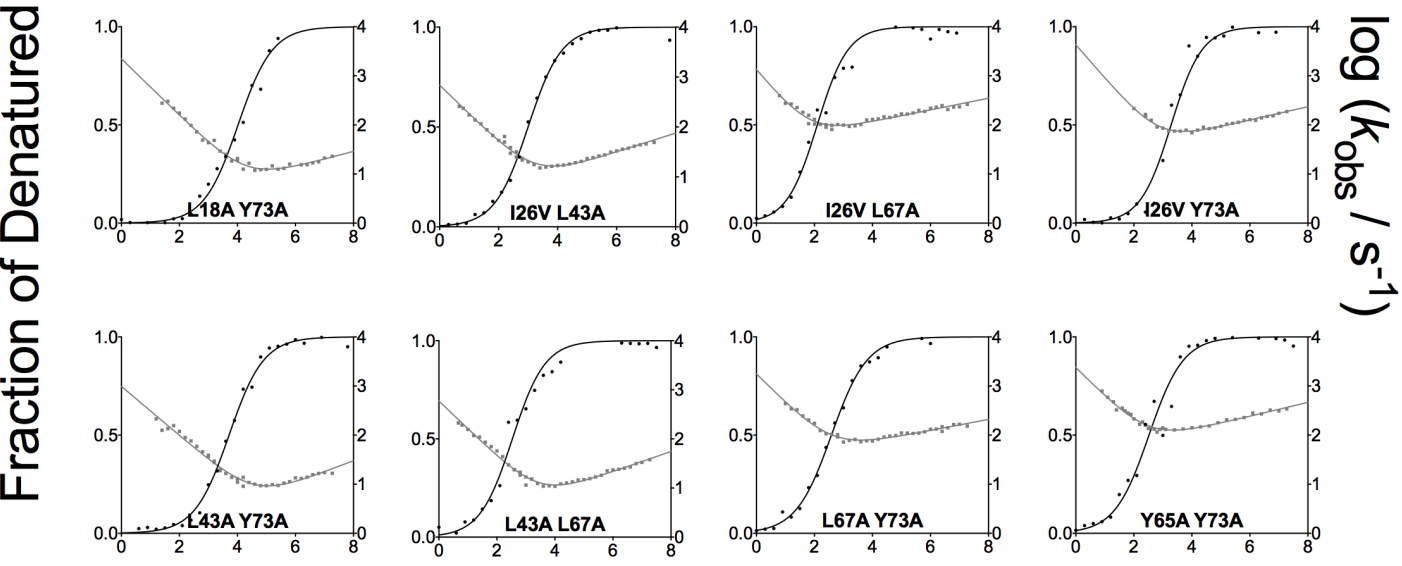

[Urea] (M)

Figure 4 

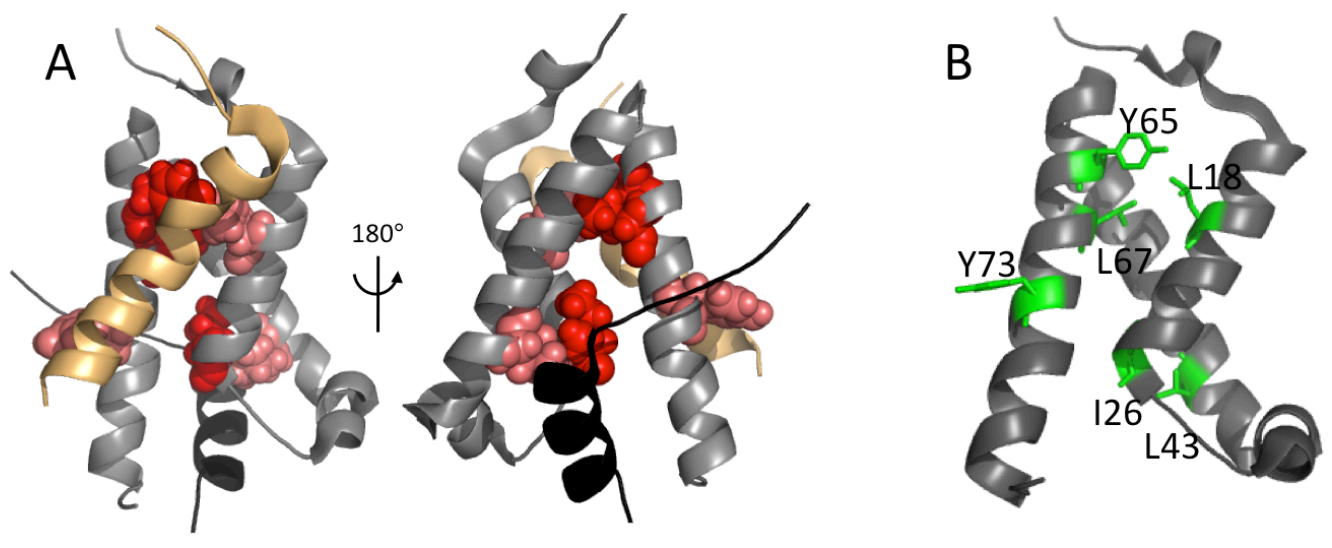

Figure 5 